\title{
Harmonic oscillator chains as Wigner Quantum Systems: periodic and fixed wall boundary conditions in $\mathfrak{g l}(1 \mid n)$ solutions.
}

\author{
S. Lievenst, N.I. Stoilovat and J. Van der Jeugt \\ Department of Applied Mathematics and Computer Science, Ghent University, \\ Krijgslaan 281-S9, B-9000 Gent, Belgium.
}

\begin{abstract}
We describe a quantum system consisting of a one-dimensional linear chain of $n$ identical harmonic oscillators coupled by a nearest neighbor interaction. Two boundary conditions are taken into account: periodic boundary conditions (where the $n$th oscillator is coupled back to the first oscillator) and fixed wall boundary conditions (where the first oscillator and the $n$th oscillator are coupled to a fixed wall). The two systems are characterized by their Hamiltonian. For their quantization, we treat these systems as Wigner Quantum Systems (WQS), allowing more solutions than just the canonical quantization solution. In this WQS approach, one is led to certain algebraic relations for operators (which are linear combinations of position and momentum operators) that should satisfy triple relations involving commutators and anticommutators. These triple relations have a solution in terms of the Lie superalgebra $\mathfrak{g l}(1 \mid n)$. We study a particular class of $\mathfrak{g l}(1 \mid n)$ representations $V(p)$, the so-called ladder representations. For these representations, we determine the spectrum of the Hamiltonian and of the position operators (for both types of boundary conditions). Furthermore, we compute the eigenvectors of the position operators in terms of stationary states. This leads to explicit expressions for position probabilities of the $n$ oscillators in the chain. An analysis of the plots of such position probability distributions gives rise to some interesting observations. In particular, the physical behavior of the system as a WQS is very much in agreement with what one would expect from the classical case, except that all physical quantities (energy, position and momentum of each oscillator) have a finite spectrum.
\end{abstract}

\footnotetext{
${ }^{\dagger}$ E-mail: Stijn.Lievens@UGent.be

${ }^{\ddagger}$ E-mail: Neli.Stoilova@UGent.be; Permanent address: Institute for Nuclear Research and Nuclear Energy, Boul. Tsarigradsko Chaussee 72, 1784 Sofia, Bulgaria

${ }^{\S}$ E-mail: Joris.VanderJeugt@UGent.be
} 


\section{Introduction}

Coupled systems describing interaction of oscillating or scattering subsystems and the corresponding operators have been widely used in classical and quantum mechanics. For example, coupled harmonic oscillators have been studied in quantum information theory, quantum optics (photonic crystals), and for describing phonons in a crystal [1-6].

In a previous paper [7], we initiated the analysis of such systems as a Wigner Quantum System (WQS) [8-10]. The system studied in [7] consists of a string or chain of $n$ identical harmonic oscillators, each having the same mass $m$ and frequency $\omega$. The position and momentum operator for the $r$ th oscillator $(r=1,2, \ldots, n)$ are given by $\hat{q}_{r}$ and $\hat{p}_{r}$; more precisely $\hat{q}_{r}$ measures the displacement of the $r$ th mass point with respect to its equilibrium position. The oscillators are coupled by some nearest neighbor coupling, represented by terms of the form $\left(\hat{q}_{r}-\hat{q}_{r+1}\right)^{2}$ in the Hamiltonian. In [7], we considered such a system with periodic boundary conditions, where the last oscillator is again coupled to the first one (i.e. $\hat{q}_{n+1}=\hat{q}_{1}$ ). In other words, the oscillators are on a circle rather than on a line. The Hamiltonian of this system will be denoted by $\hat{H}_{P}$. In the present paper we will reconsider this system, and at the same time also concentrate on a second type of boundary conditions: fixed wall boundary conditions. For this second case, the first and last oscillator are coupled to a fixed wall (i.e. $\hat{q}_{0}=\hat{q}_{n+1}=0$ ); the Hamiltonian of this second system will be denoted by $\hat{H}_{F W}$.

The original contribution of [7] consisted of the treatment of the system described by $\hat{H}_{P}$ as a Wigner Quantum System [8-10]. This quantization procedure is based upon the compatibility of Hamilton's equations and the Heisenberg equations. In this procedure, one does not require the canonical commutation relations (CCRs), but instead uses more general relations describing the before mentioned compatibility. As a consequence, one obtains different classes of solutions for the system, of which the canonical solution is only one. For a more detailed description of WQSs, see [7] and references therein. The main feature of this treatment is that the CCRs are replaced by these compatibility conditions (CCs). For the Hamiltonian $\hat{H}_{P}$, these CCs are triple relations in terms of operators $a_{r}^{ \pm}(r=1,2, \ldots, n)$, involving both commutators and anti-commutators. Herein, the operators $a_{r}^{ \pm}$are certain linear combinations of the position and momentum operators. In [7] we showed that these triple relations have a solution in terms of certain generators of the Lie superalgebra $\mathfrak{g l}(1 \mid n)[11]$. Furthermore, we considered a class of Fock representations [12] of $\mathfrak{g l}(1 \mid n)$ and analysed the energy spectrum and the position operator spectrum in these Fock representations $W(p)$. These Fock representations are rather restricted, however, and do not illustrate the features of general unitary irreducible representations [13] of $\mathfrak{g l}(1 \mid n)$ (more precisely, of its compact form $\mathfrak{u}(1 \mid n))$.

In the present paper, we consider a more general class of $\mathfrak{g l}(1 \mid n)$ representations, the so-called ladder representations $V(p)$ [13]. These representations are also easy to describe, but more importantly they show interesting properties of the physical quantities (energy spectrum, position operator spectrum) of the system, far more general than those of the Fock representations $W(p)$.

A second original contribution of this paper is that we show how also the second system (fixed wall boundary conditions) described by $\hat{H}_{F W}$ can be treated as a Wigner Quantum System. In fact, we show that at the algebraic level the two Hamiltonians give rise to the same triple relations, but with different constants. As a consequence, the two systems can be treated similarly, both having solutions in terms of the Lie superalgebra $\mathfrak{g l}(1 \mid n)$.

The contents of the paper is as follows. In Section 2 we shortly review the treatment of $\hat{H}_{P}$ as a WQS [7], and then analyse the Hamiltonian $\hat{H}_{F W}$ of the fixed wall boundary case in a similar way. As the algebraic triple relations are, up to different constants, the same, we describe the $\mathfrak{g l}(1 \mid n)$ solution in a subsection. Special attention is paid to determining the critical value for the coupling constant $c$, for which a $\mathfrak{g l}(1 \mid n)$ solution exists in the fixed wall boundary case. We also 
describe briefly the new class of representations $V(p)$, the ladder representations, and the explicit action of $\mathfrak{g l}(1 \mid n)$ generators on simple basis vectors $w(\theta ; s)$ of $V(p)$. In Section 3 we determine the spectrum of the Hamiltonians $\hat{H}_{P}$ and $\hat{H}_{F W}$ in the representations $V(p)$. Due to the fact that these Hamiltonians have a diagonal action in the current model, their spectrum is easy to determine. We compare the two cases, and discuss some aspects of degeneracy of the energy levels.

The mathematically more difficult problem is that of determining the eigenvalues and eigenvectors of the position operators $\hat{q}_{r}$, for these representations $V(p)$, since $\hat{q}_{r}$ is a general odd operator of $\mathfrak{g l}(1 \mid n)$ with a nondiagonal action on the standard basis of $V(p)$. Fortunately, we have managed to give a general treatment (and procedure) of how to construct such eigenvalue spectrum and eigenvectors for an arbitrary odd element of $\mathfrak{g l}(1 \mid n)$ in an arbitrary unitary representation in [14]. Using the techniques of [14], we manage to determine the eigenvalues of $\hat{q}_{r}$ for both systems in Section 4, and to construct the eigenvectors of $\hat{q}_{r}$ in Section 5. In fact, the eigenvalues of $\hat{q}_{r}$ for the system described by $\hat{H}_{P}$ were already given in [14] as an example of the new technique, so only those for the second system described by $\hat{H}_{F W}$ are new. We also give some plots of the spectrum of the position operators, as a function of the coupling constant $c$, and make some observations related to these plots.

Section 5 is devoted to computing position probability distributions for the two systems under consideration. Since the techniques of [14] allow us to give an explicit expansion of the normalized eigenvectors of $\hat{q}_{r}$, for any of its eigenvalues $\pm x_{K}$, in terms of the stationary states $w(\theta ; s)$, we can invert these relations and express the stationary states $w(\theta ; s)$ in terms of the eigenvectors of $\hat{q}_{r}$ (for any particular $r$ ). The square moduli of the coefficients in these expressions have the usual quantum theory interpretation as the probability of finding the $r$ th oscillator in position $\pm x_{K}$ when the system is in its stationary state $w(\theta ; s)$. We compute these probabilities analytically (for small $p$-values), and numerically for some examples. We also give a number of plots of such probability distribution functions. These yield the "spacial properties" of the oscillators. The results are quite interesting, and show some analogy of what one would expect of the system from a classical treatment. For the ground state (stationary state of lowest energy), the position with highest probability (for each oscillator) is the equilibrium position (corresponding to $x_{p}=0$ ), with strongly decreasing probability as the (discrete) eigenvalue is further away from 0 . For the most excited state (stationary state of highest energy), the position with highest probability is away from 0 (symmetrically to the left and the right). The higher the coupling constant, the further away this highest probability position. The probability of the equilibrium position is zero here. All oscillators have the same position probability distribution in the periodic boundary case, as they are all equivalent (completely symmetric on a circle). In the case of fixed wall boundary conditions, the behavior also follows the classical properties, with the first and last oscillators (those fixed to the wall) "oscillating less" compared to those away from the wall. We end the paper by some concluding remarks in Section 6.

\section{The Hamiltonian of the system: solutions as a WQS}

\subsection{System with periodic boundary conditions}

In this subsection, we briefly recall the algebraic treatment of the Hamiltonian for a system consisting of coupled harmonic oscillators, with periodic boundary conditions, treated as a Wigner Quantum System [7]. In several models [2-6] such a quantum system consisting of a linear chain of $n$ identical harmonic oscillators coupled by springs is used. The Hamiltonian of such a system is given by:

$$
\hat{H}_{P}=\sum_{r=1}^{n}\left(\frac{\hat{p}_{r}^{2}}{2 m}+\frac{m \omega^{2}}{2} \hat{q}_{r}^{2}+\frac{c m}{2}\left(\hat{q}_{r}-\hat{q}_{r+1}\right)^{2}\right) \text {, }
$$


where each oscillator has mass $m$ and frequency $\omega, \hat{q}_{r}$ and $\hat{p}_{r}$ stand for the position and momentum operator for the $r$ th oscillator (or rather, $\hat{q}_{r}$ measures the displacement of the $r$ th mass point with respect to its equilibrium position), and $c>0$ is the coupling strength. In the case of periodic boundary conditions (indicated by the index in $\hat{H}_{P}$ ), one assumes in (2.1)

$$
\hat{q}_{n+1} \equiv \hat{q}_{1} .
$$

With these periodic boundary conditions one can think of the oscillators as being located on a circle, as the last oscillator is again coupled to the first one.

In [7], it was shown that one can relax the canonical commutation relations for the operators $\hat{q}_{r}$ and $\hat{p}_{r}$, leading to a larger class of solutions for the system described by (2.1). This is known as a Wigner Quantum System approach $[9,10]$. In this approach, one imposes the compatibility of Hamilton's equations

$$
\dot{\hat{q}}_{r}=\frac{\partial \hat{H}_{P}}{\partial \hat{p}_{r}}, \quad \dot{\hat{p}}_{r}=-\frac{\partial \hat{H}_{P}}{\partial \hat{q}_{r}} \quad(r=1,2, \ldots, n)
$$

(formal derivatives) and the Heisenberg equations

$$
\dot{\hat{p}}_{r}=\frac{i}{\hbar}\left[\hat{H}_{P}, \hat{p}_{r}\right], \quad \dot{\hat{q}}_{r}=\frac{i}{\hbar}\left[\hat{H}_{P}, \hat{q}_{r}\right] \quad(r=1,2, \ldots, n)
$$

when viewed as operator equations. These compatibility conditions (CCs) read

$$
\begin{aligned}
& {\left[\hat{H}_{P}, \hat{q}_{r}\right]=-\frac{i \hbar}{m} \hat{p}_{r},} \\
& {\left[\hat{H}_{P}, \hat{p}_{r}\right]=-i \hbar c m \hat{q}_{r-1}+i \hbar m\left(\omega^{2}+2 c\right) \hat{q}_{r}-i \hbar c m \hat{q}_{r+1},}
\end{aligned}
$$

with $r \in\{1,2, \ldots, n\}, \hat{q}_{n+1}=\hat{q}_{1}$ and $\hat{q}_{0}=\hat{q}_{n}$. Under the canonical commutation relations, the CCs (2.5) and (2.6) are automatically satisfied. But the system (2.5)-(2.6), with $\hat{H}_{P}$ given by (2.1), has other interesting solutions [7].

To study these other solutions, one introduces discrete Fourier transforms of the (self-adjoint) operators $\hat{q}_{r}$ and $\hat{p}_{r}$ by

$$
\begin{aligned}
& \hat{q}_{r}=\sum_{j=1}^{n} \sqrt{\frac{\hbar}{2 m n \omega_{j}}}\left(e^{-2 \pi i j r / n} a_{j}^{+}+e^{2 \pi i j r / n} a_{j}^{-}\right), \\
& \hat{p}_{r}=\sum_{j=1}^{n} i \sqrt{\frac{m \omega_{j} \hbar}{2 n}}\left(e^{-2 \pi i j r / n} a_{j}^{+}-e^{2 \pi i j r / n} a_{j}^{-}\right),
\end{aligned}
$$

where $\omega_{j}$ are positive numbers with

$$
\omega_{j}^{2}=\omega^{2}+2 c-2 c \cos \left(\frac{2 \pi j}{n}\right)=\omega^{2}+4 c \sin ^{2}\left(\frac{\pi j}{n}\right),
$$

and $a_{j}^{ \pm}$are operators satisfying $\left(a_{j}^{ \pm}\right)^{\dagger}=a_{j}^{\mp}$. In terms of these new operators, the Hamiltonian reads $[7]$

$$
\hat{H}_{P}=\sum_{j=1}^{n} \frac{\hbar \omega_{j}}{2}\left(a_{j}^{-} a_{j}^{+}+a_{j}^{+} a_{j}^{-}\right) .
$$

Note that we no longer require the canonical commutation relations for the operators $\hat{q}_{r}$ and $\hat{p}_{r}$, so also the operators $a_{j}^{ \pm}$no longer satisfy the usual boson relations $\left[a_{j}^{ \pm}, a_{k}^{ \pm}\right]=0$ and $\left[a_{j}^{-}, a_{k}^{+}\right]=\delta_{j k}$. In the WQS approach, the relations that should be satisfied follow from (2.5)-(2.6), and read explicitly [7]:

$$
\left[\sum_{j=1}^{n} \omega_{j}\left(a_{j}^{-} a_{j}^{+}+a_{j}^{+} a_{j}^{-}\right), a_{k}^{ \pm}\right]= \pm 2 \omega_{k} a_{k}^{ \pm}, \quad(k=1,2, \ldots, n) .
$$




\subsection{System with fixed wall boundary conditions}

In this subsection we consider a similar system, again consisting of coupled harmonic oscillators, but this time with fixed wall boundary conditions. Now the Hamiltonian reads:

$$
\hat{H}_{F W}=\sum_{r=1}^{n}\left(\frac{\hat{p}_{r}^{2}}{2 m}+\frac{m \omega^{2}}{2} \hat{q}_{r}^{2}+\frac{c m}{2}\left(\hat{q}_{r}-\hat{q}_{r+1}\right)^{2}\right),
$$

with the same data as in (2.1), but

$$
\hat{q}_{0}=\hat{q}_{n+1} \equiv 0 \quad\left(\text { and } \hat{p}_{0}=\hat{p}_{n+1} \equiv 0\right) .
$$

In other words, we assume that the first and last oscillator (i.e. the oscillators numbered 1 and $n$ ) are attached to a fixed wall.

The treatment of this Hamiltonian in the WQS approach is very similar to the previous subsection. In fact, the CCs (2.5)-(2.6) remain the same (with $\hat{H}_{P}$ replaced by $\hat{H}_{F W}$, but with $\hat{q}_{0}=\hat{q}_{n+1}=0$ ). These different boundary conditions lead to a different type of transform. Instead of a discrete Fourier transform, we now use a discrete sine transform. More explicitly, we introduce the following transformations of the (self-adjoint) operators $\hat{q}_{r}$ and $\hat{p}_{r}$ :

$$
\begin{aligned}
& \hat{q}_{r}=\sum_{j=1}^{n} \sqrt{\frac{\hbar}{m(n+1) \tilde{\omega}_{j}}} \sin \left(\frac{r j \pi}{n+1}\right)\left(a_{j}^{+}+a_{j}^{-}\right), \\
& \hat{p}_{r}=i \sum_{j=1}^{n} \sqrt{\frac{m \tilde{\omega}_{j} \hbar}{n+1}} \sin \left(\frac{r j \pi}{n+1}\right)\left(a_{j}^{+}-a_{j}^{-}\right),
\end{aligned}
$$

where the $\tilde{\omega}_{j}$ are positive numbers given by

$$
\tilde{\omega}_{j}^{2}=\omega^{2}+2 c-2 c \cos \left(\frac{j \pi}{n+1}\right)=\omega^{2}+4 c \sin ^{2}\left(\frac{j \pi}{2(n+1)}\right) .
$$

The operators $a_{j}^{ \pm}$satisfy the adjointness conditions

$$
\left(a_{j}^{ \pm}\right)^{\dagger}=a_{j}^{\mp} .
$$

In terms of these new operators, the Hamiltonian (2.12) is given by:

$$
\hat{H}_{F W}=\sum_{j=1}^{n} \frac{\hbar \tilde{\omega}_{j}}{2}\left(a_{j}^{-} a_{j}^{+}+a_{j}^{+} a_{j}^{-}\right)
$$

and the compatibility conditions become

$$
\left[\sum_{j=1}^{n} \tilde{\omega}_{j}\left(a_{j}^{-} a_{j}^{+}+a_{j}^{+} a_{j}^{-}\right), a_{k}^{ \pm}\right]= \pm 2 \tilde{\omega}_{k} a_{k}^{ \pm}, \quad(k=1,2, \ldots, n) .
$$

So the algebraic expression of the Hamiltonian, and the CCs, are the same in the two cases considered, apart from the replacement $\omega_{j} \rightarrow \tilde{\omega}_{j}$. This implies that the algebraic solutions will be similar, even though the conclusions about physical properties will be different due to the different numerical values of the numbers $\omega_{j}$ and $\tilde{\omega}_{j}$. 


\subsection{The $\mathfrak{g l}(1 \mid n)$ solution}

In was shown in [7] that the triple relations (2.11) involving both anti-commutators and commutators have a solution in terms of generators of the Lie superalgebra $\mathfrak{g l}(1 \mid n)$ [11]. More explicitly, let $\mathfrak{g l}(1 \mid n)$ be the Lie superalgebra with standard basis elements $e_{j k}(j, k=0,1, \ldots, n)$ where $e_{k 0}$ and $e_{0 k}(k=1, \ldots, n)$ are odd elements $\left(\operatorname{deg} e_{0 k}=\operatorname{deg} e_{k 0}=1\right)$ and the remaining basis elements are even (having degree 0), with bracket

$$
\llbracket e_{i j}, e_{k l} \rrbracket=\delta_{j k} e_{i l}-(-1)^{\operatorname{deg}\left(e_{i j}\right) \operatorname{deg}\left(e_{k l}\right)} \delta_{i l} e_{k j},
$$

and star condition $e_{i j}^{\dagger}=e_{j i}$. Then a solution of (2.11) is provided by

$$
a_{j}^{-}=\sqrt{\frac{2 \beta_{j}}{\omega_{j}}} e_{j 0}, \quad a_{j}^{+}=\sqrt{\frac{2 \beta_{j}}{\omega_{j}}} e_{0 j} \quad(j=1, \ldots, n)
$$

where

$$
\beta_{j}=-\omega_{j}+\frac{1}{n-1} \sum_{k=1}^{n} \omega_{k}, \quad(j=1, \ldots, n) .
$$

All these numbers $\beta_{j}$ should be nonnegative. By the periodic boundary conditions, the $\beta_{j}$ 's satisfy $\beta_{n-j}=\beta_{j}$, and

$$
\beta_{1}>\beta_{2}>\cdots>\beta_{\lfloor n / 2\rfloor}, \quad \beta_{\lfloor n / 2\rfloor} \leq \beta_{\lfloor n / 2\rfloor+1}<\cdots<\beta_{n} .
$$

It was analysed in [7] that all these $\beta_{j}$ 's are indeed positive provided the coupling constant $c$ lies in a certain interval $\left[0, c_{0}\left[\right.\right.$, with $c_{0}$ some critical value depending upon $n$.

In the case of fixed wall boundary conditions, the analysis is slightly different. A solution of (2.19) is given by

$$
a_{j}^{-}=\sqrt{\frac{2 \tilde{\beta}_{j}}{\tilde{\omega}_{j}}} e_{j 0}, \quad a_{j}^{+}=\sqrt{\frac{2 \tilde{\beta}_{j}}{\tilde{\omega}_{j}}} e_{0 j} \quad(j=1, \ldots, n)
$$

where in this case

$$
\tilde{\beta}_{j}=-\tilde{\omega}_{j}+\frac{1}{n-1} \sum_{k=1}^{n} \tilde{\omega}_{k}, \quad(j=1, \ldots, n) .
$$

Again, all these numbers $\tilde{\beta}_{j}$ should be nonnegative. First, note that for $c>0$ one has that $\tilde{\omega}_{1}<\tilde{\omega}_{2}<\cdots<\tilde{\omega}_{n}$ and hence that

$$
\tilde{\beta}_{1}>\tilde{\beta}_{2}>\cdots>\tilde{\beta}_{n}
$$

Thus all $\tilde{\beta}_{j}$ are positive if and only if $\tilde{\beta}_{n}$ is positive. Secondly, for $c=0$ one has that $\tilde{\beta}_{j}=$ $\omega /(n-1)>0$, and since $\tilde{\beta}_{n}$ is a continuous function of $c$ there exist positive values of $c$ such that $\tilde{\beta}_{n}>0$. Thus, there exists (in general) a critical value $\tilde{c}_{0}$ such that each $\tilde{\beta}_{j}>0$ for $c<\tilde{c}_{0}$ and such that for $c=\tilde{c}_{0}$ one has that $\tilde{\beta}_{n}=0$. The same upper bound on the critical value $\tilde{c}_{0}$ applies as on $c_{0}$ in periodic boundary conditions case since one can mimic the proof of [7, Proposition 2]. Since for $n=2$ we have that $\tilde{\beta}_{2}=\sqrt{\omega^{2}+c}$ there are no conditions on $c$ in this case. Also for $n=3$ there are no conditions since one can verify (numerically) that in this case $\tilde{\beta}_{3}>0$. In Table 1, we compare the critical values $c_{0} / \omega^{2}$ of the periodic boundary conditions case with the critical values $\tilde{c}_{0} / \omega^{2}$. One notices that these critical values are interleaved, and that they become more and more similar as the number of oscillators $n$ increases. 


\begin{tabular}{||r|r|r||r|r|r||}
\hline$n$ & $c_{0} / \omega^{2}$ & $\tilde{c}_{0} / \omega^{2}$ & $n$ & $c_{0} / \omega^{2}$ & $\tilde{c}_{0} / \omega^{2}$ \\
\hline 4 & 0.9873724357 & 2.1108888881 & 13 & 0.10546881460 & 0.10521909714 \\
5 & 0.7500000000 & 0.7016444817 & 14 & 0.09256321610 & 0.09509684206 \\
6 & 0.3457442295 & 0.4138598334 & 15 & 0.08687882025 & 0.08675785013 \\
7 & 0.2982653656 & 0.2921798279 & 16 & 0.07814800074 & 0.07976866442 \\
8 & 0.2061705212 & 0.2254893243 & 17 & 0.07388896853 & 0.07382573538 \\
9 & 0.1851128402 & 0.1835156565 & 18 & 0.06760983697 & 0.06871018095 \\
10 & 0.1464642846 & 0.1547079900 & 19 & 0.06429500840 & 0.06426020587 \\
11 & 0.1343028683 & 0.1337254495 & 20 & 0.05957194222 & 0.06035363583 \\
12 & 0.1134651313 & 0.1177656002 & 21 & 0.05691629341 & 0.05689649085 \\
\hline
\end{tabular}

Table 1: Critical values $c_{0} / \omega^{2}$ (periodic boundary conditions) and $\tilde{c}_{0} / \omega^{2}$ (fixed wall boundary conditions).

\subsection{A class of $\mathfrak{g l}(1 \mid n)$ representations $V(p)$}

In the case of canonical commutations relations, there is essentially only one representation of the system, following from the Heisenberg-Weyl algebra satisfied by the operators $\hat{q}_{r}$ and $\hat{p}_{r}$. In the case of WQS, the properties of the systems described by the Hamiltonians $\hat{H}_{P}$ and $\hat{H}_{F W}$ depend on the $\mathfrak{g l}(1 \mid n)$ representation considered. In principle, any unitary representation of $\mathfrak{g l}(1 \mid n)$ can be taken into account [13]. A simple class of Fock representations $W(p)$ [12] was already investigated in [7], for the case of periodic boundary conditions. This class of Fock representations is easy to work with, but also rather restricted as the basis vectors involve "fermionic" variables only, see [7, (4.1)].

In this paper, we will consider a richer class of representations, the so-called ladder representations $V(p)$ [13]. These representations were considered as a special case in [14]. They are characterized by a positive integer $p$, and are finite-dimensional unitary representations atypical of type 2 [14]. A simple notation for the vectors of $V(p)$ is:

$$
w(\theta ; s) \equiv w\left(\theta ; s_{1}, s_{2}, \ldots, s_{n}\right), \quad \theta \in\{0,1\}, s_{i} \in\{0,1,2, \ldots\}, \quad \text { and } \theta+s_{1}+\cdots+s_{n}=p .
$$

Thus here the basis vectors involve one "fermionic" variable $\theta$ and $n$ "bosonic" variables $s_{i}$. In this notation the highest weight vector is $w(1 ; p-1,0, \ldots, 0)$.

The action of the $\mathfrak{g l}(1 \mid n)$ generators on the basis (2.27) is given by $(1 \leq k \leq n)[13,14]$ :

$$
\begin{aligned}
& e_{00} w(\theta ; s)=\theta w(\theta ; s), \\
& e_{k k} w(\theta ; s)=s_{k} w(\theta ; s), \\
& e_{k 0} w(\theta ; s)=\theta \sqrt{s_{k}+1} w\left(1-\theta ; s_{1}, \ldots, s_{k}+1, \ldots, s_{n}\right), \\
& e_{0 k} w(\theta ; s)=(1-\theta) \sqrt{s_{k}} w\left(1-\theta ; s_{1}, \ldots, s_{k}-1, \ldots, s_{n}\right) .
\end{aligned}
$$

From these one deduces the action of other elements $e_{k l}$. The basis $w(\theta ; s)$ of $V(p)$ is orthogonal, i.e. $\left\langle w(\theta ; s), w\left(\theta^{\prime} ; s^{\prime}\right)\right\rangle=\delta_{\theta, \theta^{\prime}} \delta_{s, s^{\prime}}$, and with respect to this inner product the action of the generating elements satisfies the conjugacy relations $e_{k 0}^{\dagger}=e_{0 k}$ and $e_{0 k}^{\dagger}=e_{k 0}$.

\section{On the spectrum of the Hamiltonians in the ladder representa- tion}

In this section, we study the spectrum of the Hamiltonians (2.1) and (2.12) in the ladder representation $V(p)$ of the $\mathfrak{g l}(1 \mid n)$ solution. Although the basis vectors of the representation are in both cases eigenvectors of the Hamiltonian, the spectrum in the two cases is quite different. 


\subsection{Energy eigenvalues in case of periodic boundary conditions}

For the periodic boundary conditions case, the Hamiltonian (2.10) is given, using (2.21), by:

$$
\hat{H}_{P}=\hbar\left(\beta e_{00}+\sum_{k=1}^{n} \beta_{k} e_{k k}\right)
$$

with $\beta=\sum_{k=1}^{n} \beta_{k}=\sum_{k=1}^{n} \omega_{k}$. Since the action of each $e_{k k}$ is diagonal in the basis $w(\theta ; s)$, see (2.28) and (2.29), this implies that each basis vector $w(\theta ; s)$ is an eigenvector of $\hat{H}_{P}$, or a stationary state. Indeed, one finds that

$$
\hat{H}_{P} w(\theta ; s)=\hbar\left(\beta \theta+\sum_{k=1}^{n} \beta_{k} s_{k}\right) w(\theta ; s)=\hbar E_{\theta, s} w(\theta ; s) .
$$

When $c=0$, one has that $\beta_{k}=\omega /(n-1)$ and $\beta=\omega n /(n-1)$, so in this case there are only two eigenvalues namely

$$
E_{0, s}=\frac{\omega p}{n-1} \quad \text { and } \quad E_{1, s}=\frac{\omega p}{n-1}+\omega
$$

with multiplicities

$$
\left(\begin{array}{c}
p+n-1 \\
n-1
\end{array}\right) \text { and }\left(\begin{array}{c}
p+n-2 \\
n-1
\end{array}\right)
$$

respectively.

When $c>0$, these two levels each split into a number of energy levels with lower degeneracies. Recall that $\beta_{k}=\beta_{n-k}$. So, when $n=2 r$ is even, one can rewrite $E_{\theta, s}$ as follows:

$$
E_{\theta, s}=\beta \theta+\sum_{k=1}^{r-1} \beta_{k}\left(s_{k}+s_{n-k}\right)+\beta_{r} s_{r}+\beta_{n} s_{n} .
$$

It is then clear that any basis vector $w\left(\theta ; s^{\prime}\right)$ for which $s_{k}^{\prime}+s_{n-k}^{\prime}=s_{k}+s_{n-k}(k=1, \ldots, n-1)$ yields the same $\hat{H}_{P}$ eigenvalue, independent of the value of $c$, i.e. for such $\theta$ and $s^{\prime}$ one has that $E_{\theta, s}=E_{\theta, s^{\prime}}$. Alternatively, one can say that for a fixed value of $\theta, E_{\theta, s}$ is completely determined by

$$
\left(s_{1}+s_{n-1}, s_{2}+s_{n-2}, \ldots, s_{r-1}+s_{n-r+1}, s_{r}, s_{n}\right),
$$

which is a composition of $p-\theta$ into $r+1$ parts. In the same way one sees that when $n=2 r+1$ is odd, $E_{\theta, s}$ with $\theta$ fixed, is determined by

$$
\left(s_{1}+s_{n-1}, s_{2}+s_{n-2}, \ldots, s_{r-1}+s_{n-r+1}, s_{r}+s_{r+1}, s_{n}\right),
$$

For $0<c \leq c_{0}$ and $n=2 r$ or $n=2 r+1$, the number of different energy levels is thus in general given by

$$
\left(\begin{array}{c}
p+r \\
p
\end{array}\right)+\left(\begin{array}{c}
p+r-1 \\
p-1
\end{array}\right)
$$

One can also say something about the degeneracy of an individual energy level $\hbar E_{\theta, s}$. The number of compositions of an integer $N$ in two parts is $N+1$. Using this fact and (3.2) or (3.3) one sees that the degeneracy of $\hbar E_{\theta, s}$ is at least:

$$
\prod_{k=1}^{\lfloor(n-1) / 2\rfloor}\left(s_{k}+s_{n-k}+1\right) .
$$


Figure 1 shows two spectra of $\hat{H}_{P}$ for $n=4$ and $n=5$ with $p=2$ in both cases. These figures confirm our findings: in general for $0<c \leq c_{0}$ there are

$$
\left(\begin{array}{c}
2+2 \\
2
\end{array}\right)+\left(\begin{array}{c}
2+2-1 \\
1
\end{array}\right)=6+3=9
$$

different energy levels. For $n=4$, the energy level $\hbar E_{\theta, s}$ with threefold degeneracy is given by

$$
E_{0 ; 2,0,0,0}=E_{0 ; 0,0,2,0}=E_{0 ; 1,0,1,0}
$$

whereas the fourfold degenerate energy level for $n=5$ is determined by

$$
E_{0 ; 1,1,0,0,0}=E_{0 ; 1,0,1,0,0}=E_{0 ; 0,1,0,1,0}=E_{0 ; 0,0,1,1,0} .
$$

However, as can be seen from the figure, for particular values of $c$ it may happen that the multiplicity of some eigenvalues is greater than stated in (3.5) (and hence the number of different energy levels is smaller than (3.4)). It is infeasible to obtain analytical expressions for the particular values of $c$ for which this will happen.

Now, we briefly turn our attention to the minimal and maximal energy eigenvalues. For $c>0$, the values $\beta_{k}$ satisfy the following inequalities:

$$
\beta_{1}>\beta_{2}>\cdots>\beta_{r}, \quad \beta_{r} \leq \beta_{r+1}<\cdots<\beta_{n} \quad \text { and } \quad \beta_{1}<\beta_{n}
$$

and equality between $\beta_{r}$ and $\beta_{r+1}$ only occurs when $n$ is odd. From this, it immediately follows that the maximal eigenvalue of the Hamiltonian in the ladder representation $V(p)$ is given by $\hbar\left(\beta+(p-1) \beta_{n}\right)$, and the corresponding eigenvector is $w(\theta, s)$ with $\theta=1$ and $s_{j}=\delta_{j, n}(p-1)$. It is also immediately clear that this eigenvalue is nondegenerate. The minimum eigenvalue is given by $\hbar p \beta_{r}$. An eigenvector is given by $w(\theta ; s)$ with $\theta=0$ and $s_{j}=\delta_{j, r} p$. When $n$ is even this eigenvalue is nondegenerate, but when $n$ is odd it is $(p+1)$-fold degenerate, since $p+1$ is the number of compositions of $p$ into two parts. Note that the minimal eigenvalue approaches 0 as $c$ tends to $c_{0}$. This is also illustrated by Figure 1]

\subsection{Energy eigenvalues in case of fixed wall boundary conditions}

In the case with fixed wall boundary conditions the Hamiltonian (2.18) becomes, using (2.24),

$$
\hat{H}_{F W}=\hbar\left(\tilde{\beta} e_{00}+\sum_{k=1}^{n} \tilde{\beta}_{k} e_{k k}\right)
$$

with $\tilde{\beta}=\sum_{k=1}^{n} \tilde{\beta}_{k}$; so clearly one has that the basis vectors of the representation are eigenvectors:

$$
\hat{H}_{F W} w(\theta ; s)=\hbar\left(\tilde{\beta} \theta+\sum_{k=1}^{n} \tilde{\beta}_{k} s_{k}\right) w(\theta ; s)=\hbar \tilde{E}_{\theta, s} w(\theta ; s) .
$$

The analysis of these eigenvalues turns out to be easier than in the previous case, because the $\tilde{\beta}_{j}$ 's do not satisfy any symmetry relations, but only the inequalities (2.26). For $c=0$ one recovers the same two energy values and degeneracies as before, but in general for $c>0$, the energy levels are nondegenerate since the $\tilde{\beta}_{k}$ do not show any symmetry. Figure 2 illustrates this fact.

In this case, the maximal eigenvalue of $\hat{H}_{F W}$ is $\hbar\left(\tilde{\beta}+(p-1) \tilde{\beta}_{1}\right)$, while the minimal eigenvalue is given by $\hbar p \tilde{\beta}_{n}$. This follows immediately from the inequalities (2.26). Of course, these eigenvalues are always nondegenerate. 
(a) $n=4$

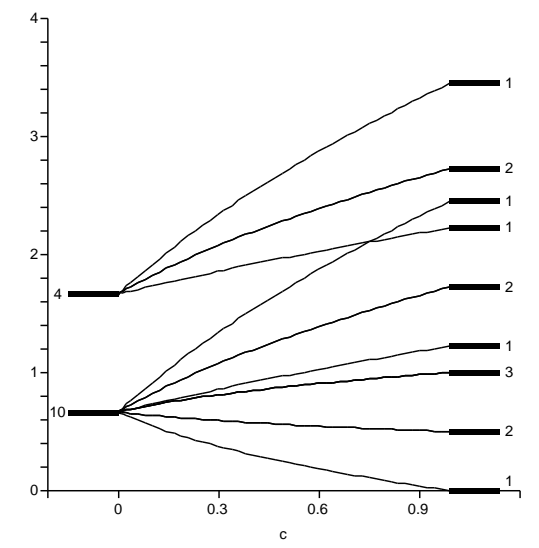

(b) $n=5$

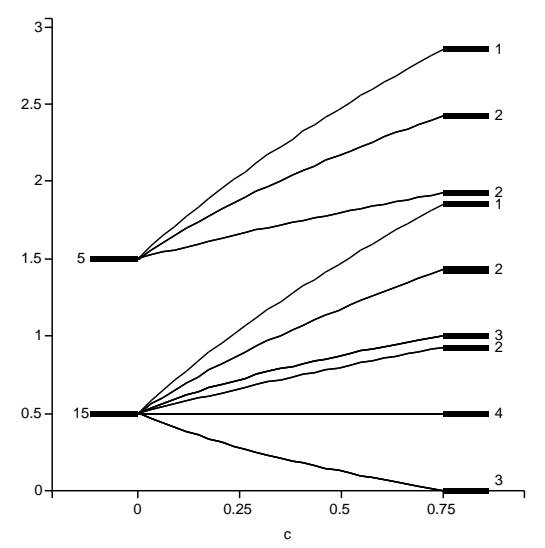

Figure 1: (a) The energy levels of the quantum system with periodic boundary conditions for $n=4$ in the representation $V(p)$ with $p=2$ and $\hbar=\omega=1 ; c$ ranges from 0 to $c_{0}$. The vertical axis gives the energy values and the numbers next to the levels refer to the multiplicity. When $c=0$ there are only two energy levels with multiplicities 10 and 4 . When $0<c<c_{0}$ there are (in general) 9 energy levels with multiplicity 1,2 or 3 . (b) The same illustration for $n=5$. Note that since $\lfloor 4 / 2\rfloor=\lfloor 5 / 2\rfloor$ the number of different energy levels is (in general) unchanged for $0<c<c_{0}$.

(a) $n=4$

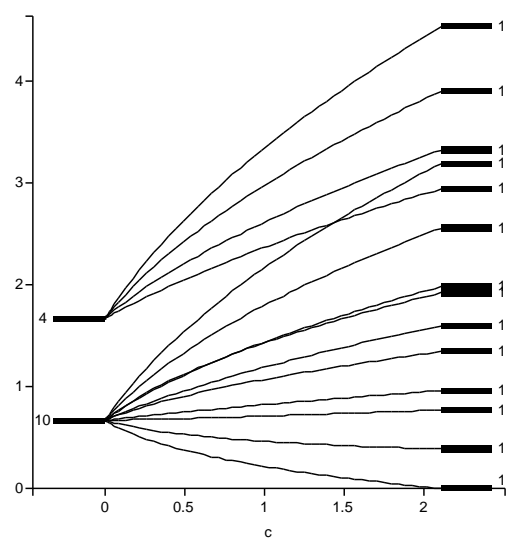

(b) $n=5$

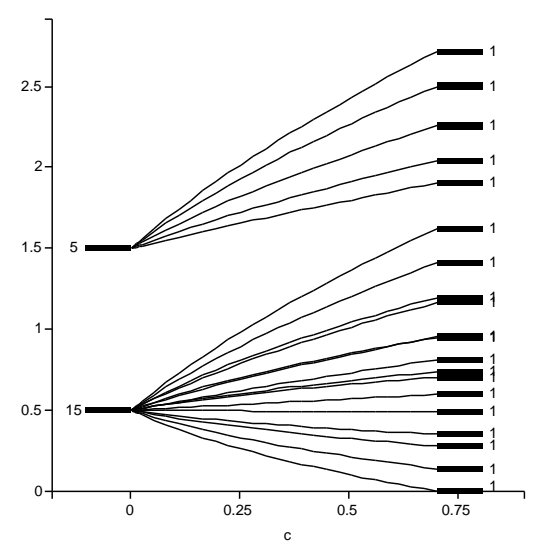

Figure 2: (a) The energy levels of the quantum system with fixed wall boundary conditions for $n=4$ in the representation $V(p)$ with $p=2$ and $\hbar=\omega=1 ; c$ ranges from 0 to $\tilde{c}_{0}$. The vertical axis gives the energy values and the numbers next to the levels refer to the multiplicity. When $c=0$ one recovers the results of the periodic boundary case. When $0<c<\tilde{c}_{0}$ however, there are (in general) 14 energy levels each with multiplicity 1 . (b) The same illustration for $n=5$, but now there are 20 nondegenerate energy levels. 


\section{On the eigenvalues and eigenvectors of the position operators}

We now turn to the study of the spectrum of the position operators $\hat{q}_{r}$, which are, under the solutions (2.21) or (2.24), quite arbitrary odd elements of the Lie superalgebra $\mathfrak{g l}(1 \mid n)$. This is precisely the topic of [14] where the eigenvalues (and eigenvectors) of the position operators $\hat{q}_{r}$ were determined for arbitrary unitary irreducible representations of the Lie superalgebra $\mathfrak{g l}(1 \mid n)$. Thus the method developed there will be applied both to the case of periodic and fixed wall boundary conditions.

\subsection{Position eigenvalues in the case of periodic boundary conditions}

The position operator $\hat{q}_{r}$, given by (2.7), can be written as

$$
\begin{aligned}
\hat{q}_{r} & =\sqrt{\frac{\hbar}{m n}} \sum_{j=1}^{n}\left(\gamma_{j} e^{2 \pi i j r / n} e_{j 0}+\gamma_{j} e^{-2 \pi i j r / n} e_{0 j}\right) \\
& =\sqrt{\frac{\hbar \gamma}{m n}}\left(E_{n 0}^{(r)}+E_{0 n}^{(r)}\right),
\end{aligned}
$$

where we use the notation

$$
\gamma_{j}=\sqrt{\beta_{j}} / \omega_{j} \quad(j=1, \ldots, n) \text { and } \gamma=\gamma_{1}^{2}+\cdots+\gamma_{n}^{2} .
$$

The odd $\mathfrak{g l}(1 \mid n)$ operators

$$
E_{n 0}^{(r)}=\frac{1}{\sqrt{\gamma}} \sum_{j=1}^{n} \gamma_{j} e^{2 \pi i j r / n} e_{j 0}=\sum_{j=1}^{n} U_{n j}^{(r)} e_{j 0}, \quad E_{0 n}^{(r)}=\sum_{j=1}^{n} U_{n j}^{(r)^{*}} e_{0 j}
$$

are part of a more general set of operators

$$
E_{j 0}^{(r)}=\sum_{l=1}^{n} U_{j l}^{(r)} e_{l 0} \text { and } E_{0 j}^{(r)}=\sum_{l=1}^{n} U_{j l}^{(r)^{*}} e_{0 l} \quad(1 \leq j \leq n),
$$

where $U=\left(U_{j l}^{(r)}\right)_{1 \leq j \leq n, 1 \leq l \leq n}$ is a unitary $n \times n$ matrix determined by the coefficients in (4.4) and in

$$
E_{j 0}^{(r)}=\frac{1}{\sqrt{\frac{1}{\gamma_{1}^{2}+\cdots+\gamma_{j}^{2}}+\frac{1}{\gamma_{j+1}^{2}}}}\left(\sum_{l=1}^{j} \frac{e^{2 \pi i r l / n}}{\gamma_{1}^{2}+\cdots+\gamma_{j}^{2}} \gamma_{l} e_{l 0}-\frac{1}{\gamma_{j+1}} e^{2 \pi i r(j+1) / n} e_{j+1,0}\right)
$$

for $j=1,2, \ldots, n-1$. The new operators $E_{j 0}^{(r)}$ and $E_{0 j}^{(r)}$ satisfy the same defining relations as the elements $e_{j 0}$ and $e_{0 j}$; in other words, they generate the Lie superalgebra $\mathfrak{s l}(1 \mid n)$ [14]. Thanks to the introduction of these new operators, one can determine the eigenvalues and eigenvectors of the position operators $\hat{q}_{r}$. This follows from the observation that (4.2) is essentially an element of $\mathfrak{g l}(1 \mid 1)$ in the decomposition $\mathfrak{g l}(1 \mid n) \rightarrow \mathfrak{g l}(1 \mid 1) \oplus \mathfrak{g l}(n-1)$. The following result was obtained in [14]:

Proposition 1 In the representation $V(p)$, all operators $\hat{q}_{r}(r=1,2, \ldots, n)$ have the same spectrum. The operator $\hat{q}_{r}$ has $2 p+1$ distinct eigenvalues given by

$$
\pm x_{K}= \pm \sqrt{\frac{\hbar \gamma}{m n}(p-K)}, \quad K=0,1, \ldots, p .
$$

The multiplicity of the eigenvalue $\pm x_{K}$ is $\left(\begin{array}{c}n-2+K \\ K\end{array}\right)$. 
Let us also briefly describe the corresponding orthonormal eigenvectors $\psi_{r, \pm x_{K}, t}$ (with $t$ a multiplicity label) corresponding to the eigenvalue $\pm x_{K}$ (see [14] for the details). For $K \neq p$, one has:

$$
\psi_{r, \pm x_{K}, t}=\frac{1}{\sqrt{2}} v\left(1 ; t_{1}, \ldots, t_{n-1}, p-1-K\right) \pm \frac{1}{\sqrt{2}} v\left(0 ; t_{1}, \ldots, t_{n-1}, p-K\right),
$$

where $t_{1}+\cdots+t_{n-1}=K$. For the eigenvalue $0(K=p)$, the eigenvectors read

$$
\psi_{r, 0, t}=v\left(0 ; t_{1}, \ldots, t_{n-1}, 0\right), \quad t_{1}+\cdots+t_{n-1}=p .
$$

We still need to describe the vectors $v(\theta ; t)$ of $V(p)$ in terms of the basis vectors $w(\theta ; s)$. Essentially,

the vectors $v(\theta ; t)$ are chosen in such a way that the action of the "new" $\mathfrak{g l}(1 \mid n)$ elements $E_{j k}^{(r)}$ on $v(\theta ; t)$ are the same as the action of the "old" $\mathfrak{g l}(1 \mid n)$ elements $e_{j k}$ on $w(\theta ; s)$. For the new highest weight vector, one has:

$$
\begin{aligned}
& v(1 ; p-1,0, \ldots, 0)= \frac{1}{\left(\gamma_{1}^{2}+\gamma_{2}^{2}\right)^{(p-1) / 2}} \sum_{u=0}^{p-1}(-1)^{u} e^{-2 \pi i r u / n} \sqrt{\left(\begin{array}{c}
p-1 \\
u
\end{array}\right)} \\
& \times \gamma_{1}^{p-1-u} \gamma_{2}^{u} w(1 ; u, p-1-u, 0, \ldots, 0) .
\end{aligned}
$$

The remaining vectors $v(\theta ; t)$ are given by

$$
\begin{aligned}
v\left(\phi ; t_{1}, \ldots, t_{n}\right) & =\frac{1}{\sqrt{N}}\left(E_{n, n-1}^{(r)}\right)^{p-\phi-\sum_{j=1}^{n-1} t_{j}}\left(E_{n-1, n-2}^{(r)}\right)^{p-\phi-\sum_{j=1}^{n-2} t_{j}} \ldots \\
& \cdots\left(E_{32}^{(r)}\right)^{p-\phi-\sum_{j=1}^{2} t_{j}}\left(E_{21}^{(r)}\right)^{p-\phi-\sum_{j=1}^{1} t_{j}}\left(E_{10}^{(r)}\right)^{1-\phi} v(1 ; p-1,0, \ldots, 0) ; \\
N & =p^{1-\phi} \prod_{k=1}^{n-1}\left(p-\phi-\sum_{j=1}^{k} t_{j}\right) !\left(t_{k}+1\right)_{p-\phi-\sum_{j=1}^{k} t_{j}},
\end{aligned}
$$

with $(a)_{n}=a(a+1) \cdots(a+n-1)$ the rising factorial symbol.

Some specific properties of the spectrum of $\hat{q}_{r}$ will be considered together with those for the fixed wall boundary conditions, in the next subsection. The expressions for the eigenvectors will be used in the following section, where position probability distributions are studied.

\subsection{Position eigenvalues in the case of fixed wall boundary conditions}

The formal part of the analysis will be rather similar to that of the previous case of periodic boundary conditions. But the outcome will show one major difference: the spectrum of $\hat{q}_{r}$ is now dependent (albeit in a simple way) on the position $r$ of the oscillator in the chain. This result is completely in accordance with physical intuition as the oscillators in the chain are clearly no longer equivalent, since we have two distinguished (and equivalent) oscillators which mark the beginning and the end of the chain.

Under the solution (2.24) of (2.19), the position operator $\hat{q}_{r}$, which is given by (2.14), becomes

$$
\begin{aligned}
\hat{q}_{r} & =\sqrt{\frac{2 \hbar}{m(n+1)}} \sum_{j=1}^{n}\left(\sin \left(\frac{r j \pi}{n+1}\right) \tilde{\gamma}_{j} e_{j 0}+\sin \left(\frac{r j \pi}{n+1}\right) \tilde{\gamma}_{j} e_{0 j}\right) \\
& =\sqrt{\frac{2 \hbar N_{r}^{2}}{m(n+1)}}\left(\tilde{E}_{n 0}^{(r)}+\tilde{E}_{0 n}^{(r)}\right) .
\end{aligned}
$$

Here, we used the abbreviations

$$
\tilde{\gamma}_{j}=\frac{\sqrt{\tilde{\beta}_{j}}}{\tilde{\omega}_{j}} \text { and } N_{r}^{2}=\sum_{j=1}^{n} \sin ^{2}\left(\frac{r j \pi}{n+1}\right) \tilde{\gamma}_{j}^{2} .
$$


Comparing with (4.2), it is clear that $\tilde{E}_{n 0}^{(r)}$ is obtained from $E_{n 0}^{(n)}$ by performing the substitutions

$$
\gamma_{j} \leadsto \tilde{\gamma}_{j} \sin \left(\frac{r j \pi}{n+1}\right) \quad(j=1, \ldots, n) .
$$

The same substitution can be used for $E_{j 0}^{(n)} \rightarrow \tilde{E}_{j 0}^{(r)}$ and $E_{0 j}^{(n)} \rightarrow \tilde{E}_{0 j}^{(r)}$. Then the analysis of the eigenvalues and eigenvectors of $\hat{q}_{r}$ is determined by the same technique as in the previous subsection. In particular:

Proposition 2 In the representation $V(p)$, the operators $\hat{q}_{r}(r=1,2, \ldots, n)$ have a spectrum depending upon $r$. The operator $\hat{q}_{r}$ has $2 p+1$ distinct eigenvalues given by

$$
\pm x_{K}= \pm \sqrt{\frac{2 \hbar N_{r}^{2}}{m(n+1)}(p-K)}, \quad K=0,1, \ldots, p
$$

The multiplicity of the eigenvalue $\pm x_{K}$ is $\left(\begin{array}{c}n-2+K \\ K\end{array}\right)$.

The corresponding orthonormal eigenvectors $\psi_{r, \pm x_{K}, t}$ are given by the same expressions (4.8)(4.9), where one should use the replacement (4.16) in (4.10) (with $r=n$ so that the complex exponential reduces to 1 ) and $E_{j k}^{(n)} \rightarrow \tilde{E}_{j k}^{(r)}$ in (4.11).

Although the spectrum of $\hat{q}_{r}$ is now dependent on the position of the oscillator in the chain (i.e. on $r$ ) through the constant $N_{r}$ in (4.17), one still has the expected symmetry that the spectra of $\hat{q}_{r}$ and $\hat{q}_{n-r+1}$ coincide, since $N_{r}^{2}=N_{n-r+1}^{2}$.

The dependence on $r$ of the spectrum of $\hat{q}_{r}$ is completely determined by $N_{r}$, which on its turn depends on the coupling constant $c$. In Figure 3, we will plot some of these eigenvalues as a function of $c$. For these plots, we use $m=\hbar=\omega=1$, and choose $K=p-1$ (then the eigenvalue expression is independent of $p$ ). We plot the cases of periodic boundary conditions and of fixed wall boundary conditions in two different figures. Thus for periodic boundary conditions, we plot the value $\sqrt{\frac{\gamma}{n}}$ as a function of $c$; for fixed wall boundary conditions, we plot the values $\sqrt{\frac{2}{n+1}} N_{r}$ as a function of $c$.

This figure, together with other numerical experiments, suggests the following about the range of the spectrum, independent of any measurement probabilities:

- When the coupling constant $c$ is fixed one has that $N_{1} \leq N_{2} \leq \cdots \leq N_{\left\lceil\frac{n}{2}\right\rceil}$. This means that the spectrum of oscillators close to the wall is more centered around their equilibrium positions than for oscillators in the middle of the chain. This is an intuitively clear result as oscillators in the middle of the chain do not "feel" the walls as much as oscillators close to the walls do.

- Viewed as a function of $c, N_{1}$ is decreasing. When the coupling constant $c$ increases, it is clear that the movements of the first oscillator will become more restricted, resulting in a spectrum closer to its equilibrium position.

- The spectra of position operators associated with oscillators in the middle of the chain seem to become very similar to one another (as $n$ increases). This is in line with intuition as these oscillators are more or less equivalent with respect to their distance to the wall. Related to this fact one sees that the spectrum of an arbitrary position operator associated with an oscillator from a chain with periodic boundary conditions is also very similar to the spectrum of an oscillator in the middle of the chain with fixed wall boundary conditions. 

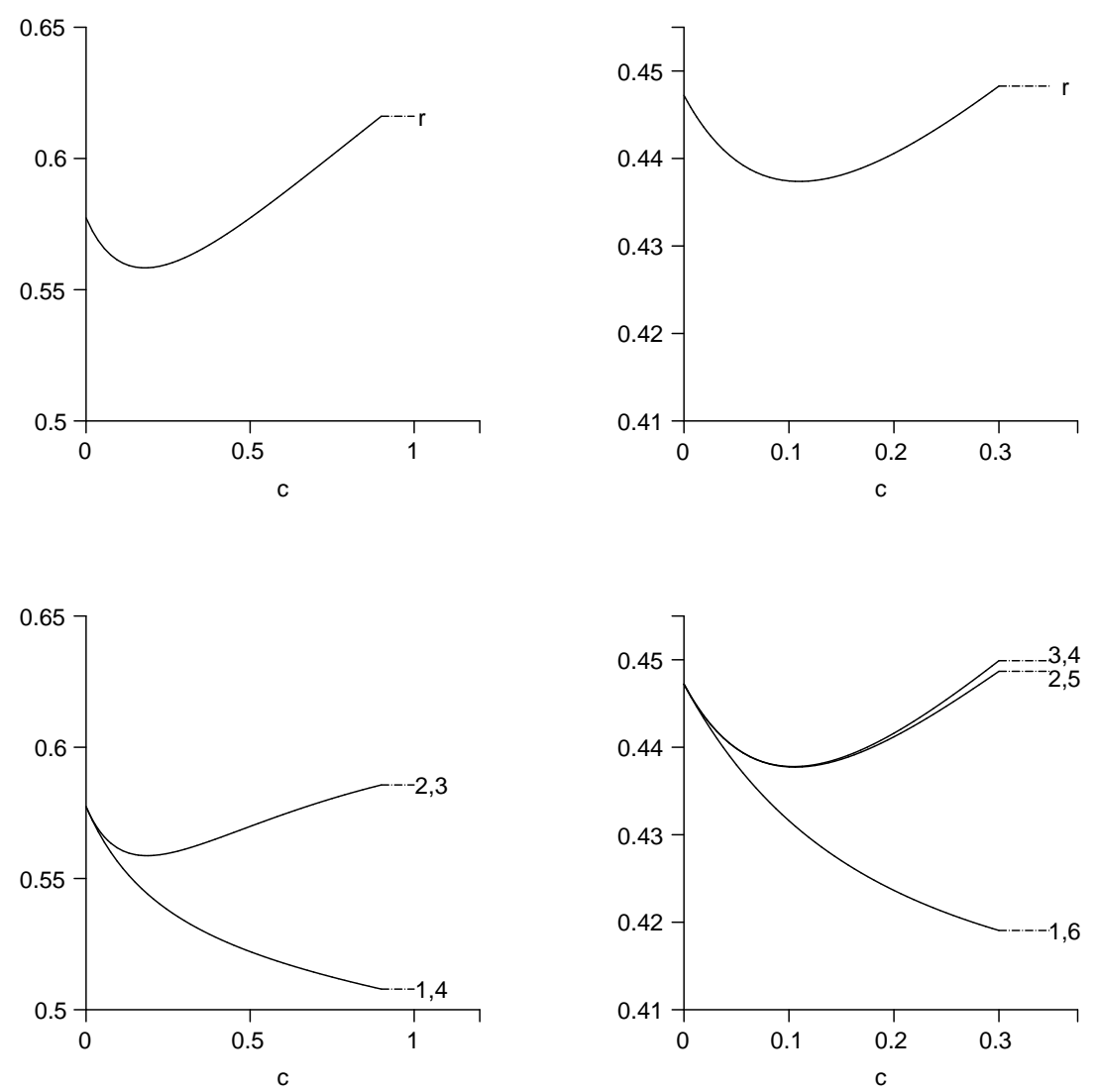

Figure 3: Indication of the spectrum of various position operators for the two chains, as a function of the coupling constant $c$ and with $m=\hbar=\omega=1$. The horizontal axis in these figures represents $c$. In the left column $n=4$, while in the right column $n=6$. In the top row $\sqrt{\gamma / n}$, i.e. the eigenvalue for an arbitray position operator $(r=1, \ldots, n)$ in a chain with periodic boundary conditions with $K=p-1$, is shown. The figures in the second row show $\sqrt{\frac{2}{n+1}} N_{r}$, with $r$ indicated next to the graph. In other words, they give the eigenvalue of the position operators in a chain with fixed wall boundary conditions (again with $K=p-1$ ). 
- For oscillators other than the first and the last, at first the spectrum moves closer to the equilibrium positions, but then seems to widen again. This effect could be related to an increasing effect of "collective" motions (lattice vibrations) as the coupling constant becomes larger.

In the above figures, we have concentrated on a particular $K$-value, i.e. on a particular eigenvalue. Of course, the complete spectrum of each $\hat{q}_{r}$ operator is easy to describe: it is simply a constant times $\pm \sqrt{p-K}, K=0,1, \ldots, p$. This is a simple distribution that will be depicted in the figures of the following section.

\section{Position probability distributions in the ladder representations}

In this section, we shall establish some facts about the position probability distributions of the two systems. It is a well known fact (postulate) of quantum mechanics that when measuring an observable, the measurement always yields an eigenvalue of the (self-adjoint) operator associated with that observable. The probability of measuring a certain eigenvalue when the system is in a certain state is determined by the expansion of that state in terms of (orthonormal) eigenvectors of the operator at hand.

We now assume that our system is in a stationary state $w(\theta ; s)$ and we wish to determine the probabilities of obtaining the different eigenvalues $x_{K}$ of an operator $\hat{q}_{r}$. In the ladder representation $V(p)$, the operator $\hat{q}_{r}$ has $2 p+1$ distinct eigenvalues $\pm x_{K}= \pm \sqrt{A_{r}(p-K)}$, where $0 \leq K \leq p$ and with $A_{r}$ a constant that in the case of periodic boundary conditions is independent of $r$, but does depend on $r$ in the case of fixed wall boundary conditions. The multiplicity of the eigenvalue $\pm x_{K}$ is $\left(\begin{array}{c}n-2+K \\ K\end{array}\right)$, and the orthonormal eigenvectors $\psi_{r, \pm x_{K}, t}$ (with $t$ a multiplicity label) have been given in the previous section. Each eigenvector $\psi_{r, \pm x_{K}, t}$ can be expanded in terms of the stationary states $w(\theta ; s)$ :

$$
\psi_{r, \pm x_{K}, t}=\sum_{\theta, s} C_{r, \pm x_{K}, t}^{\theta, s} w(\theta ; s) .
$$

Using (for the case of periodic boundary conditions) equations (4.10) and (4.11) the coefficients $C_{r, \pm x_{K}, t}^{\theta, s}$ in this expansion can be computed explicitly (the same holds of course for the case of fixed wall boundary conditions). Using orthonormality of the eigenvectors and the basis vectors, we immediately have that

$$
w(\theta ; s)=\sum_{K} \sum_{t_{1}+\cdots+t_{n-1}=K}\left(C_{r, \pm x_{K}, t}^{\theta, s}\right)^{*} \psi_{r, \pm x_{K}, t} .
$$

When the quantum system is in the fixed stationary state $w(\theta, s)$, the probability of measuring for $\hat{q}_{r}$ the eigenvalue $\pm x_{K}$ is given by:

$$
P\left(\theta, s, r, \pm x_{K}\right)=\sum_{t_{1}+\cdots+t_{n-1}=K}\left|C_{r, \pm x_{K}, t}^{\theta, s}\right|^{2} .
$$

From (4.8) it is immediately clear that

$$
P\left(\theta, s, r, x_{K}\right)=P\left(\theta, s, r,-x_{K}\right) .
$$

When $p=1$, one can determine explicit expressions for the vectors $v(\phi ; t)$. In this case when $\phi=0$ and there is exactly one $t_{j}=1$, we introduce the shorthand notation $v\left(0 ; 1^{j}\right)$ for this vector. In the case of periodic boundary conditions, the position probabilities do not depend on the position 
$r$ of the oscillator in the chain, so we work with $r=n$ so that all complex exponentials occurring in the various expressions reduce to 1 . In this case, the highest weight vector is given by

$$
v(1 ; 0, \ldots, 0)=w(1 ; 0, \ldots, 0),
$$

and using induction on $k,\left(\right.$ since $\left.v\left(0 ; 1^{k+1}\right)=E_{k+1, k}^{(n)} v\left(0 ; 1^{k}\right)\right)$ one can prove that

$$
\begin{aligned}
& v\left(0 ; 1^{k}\right)=\frac{1}{\sqrt{\gamma_{1}^{2}+\cdots+\gamma_{k+1}^{2}}}\left(\sum_{j=1}^{k} \frac{\gamma_{j} \gamma_{k+1}}{\sqrt{\gamma_{1}^{2}+\cdots+\gamma_{k}^{2}}} w\left(0 ; 1^{j}\right)-\sqrt{\gamma_{1}^{2}+\cdots+\gamma_{k}^{2}} w\left(0 ; 1^{k+1}\right)\right), \quad \text { when } k \neq n \\
& v\left(0 ; 1^{n}\right)=\frac{1}{\sqrt{\gamma_{1}^{2}+\cdots+\gamma_{n}^{2}}} \sum_{j=1}^{n} \gamma_{j} w\left(0 ; 1^{j}\right) .
\end{aligned}
$$

We can now immediately give the expansion of the eigenvectors of $\hat{q}_{n}$ in terms of the basis vectors $w(\theta ; s)$ :

$$
\begin{aligned}
\psi_{n, \pm x_{0}, t} & =\frac{1}{\sqrt{2}} w(1 ; 0, \ldots, 0) \pm \frac{1}{\sqrt{2 \gamma}} \sum_{j=1}^{n} \gamma_{j} w\left(0 ; 1^{j}\right), \quad t=(\overbrace{0, \ldots, 0}^{n-1}) . \\
\psi_{n, x_{1}, t} & =\frac{1}{\sqrt{\gamma_{1}^{2}+\cdots+\gamma_{k+1}^{2}}}\left(\sum_{j=1}^{k} \frac{\gamma_{j} \gamma_{k+1}}{\sqrt{\gamma_{1}^{2}+\cdots+\gamma_{k}^{2}}} w\left(0 ; 1^{j}\right)-\sqrt{\gamma_{1}^{2}+\cdots+\gamma_{k}^{2}} w\left(0 ; 1^{k+1}\right)\right), \quad t=1^{k},
\end{aligned}
$$

with $1 \leq k \leq n-1$. From this and (5.1) it immediately follows that

$$
P\left(\theta, s, r, x_{0}\right)= \begin{cases}\frac{1}{2} \frac{\gamma_{k}^{2}}{\gamma} & \text { when } \theta=0, s_{k}=1 \\ \frac{1}{2} & \text { when } \theta=1\end{cases}
$$

and hence

$$
P\left(\theta, s, r, x_{1}\right)= \begin{cases}\frac{\gamma-\gamma_{k}^{2}}{\gamma} & \text { when } \theta=0, s_{k}=1 \\ 0 & \text { when } \theta=1 .\end{cases}
$$

Also for $p=2$ we have been able to determine the position probabilities. They are as follows:

$$
\begin{gathered}
P\left(\theta, s, r, x_{0}\right)= \begin{cases}\frac{1}{2} \frac{\gamma_{k}^{4}}{\gamma^{2}} & \text { when } \theta=0, s_{k}=2 \\
\frac{\gamma_{k}^{2} \gamma_{l}^{2}}{\gamma^{2}} & \text { when } \theta=0, s_{k}=s_{l}=1 \\
\frac{1}{2} \frac{\gamma_{k}^{2}}{\gamma} & \text { when } \theta=1, s_{k}=1,\end{cases} \\
P\left(\theta, s, r, x_{1}\right)= \begin{cases}\frac{\gamma_{k}^{2}\left(\gamma-\gamma_{k}^{2}\right)}{\gamma^{2}} & \text { when } \theta=0, s_{k}=2 \\
\frac{1}{2} \frac{\left(\gamma-\gamma_{k}^{2}-\gamma_{l}^{2}\right)\left(\gamma_{k}^{2}+\gamma_{l}^{2}\right)+\left(\gamma_{k}^{2}-\gamma_{l}^{2}\right)^{2}}{\gamma^{2}} & \text { when } \theta=0, s_{k}=s_{l}=1 \\
\frac{1}{2} \frac{\gamma-\gamma_{k}^{2}}{\gamma} & \text { when } \theta=1, s_{k}=1,\end{cases}
\end{gathered}
$$

and

$$
P\left(\theta, s, r, x_{2}\right)= \begin{cases}\frac{\left(\gamma-\gamma_{k}^{2}\right)^{2}}{\gamma^{2}} & \text { when } \theta=0, s_{k}=2 \\ \frac{\left(\gamma-\gamma_{k}^{2}-\gamma_{l}^{2}\right) \gamma+2 \gamma_{k}^{2} \gamma_{l}^{2}}{\gamma^{2}} & \text { when } \theta=0, s_{k}=s_{l}=1 \\ 0 & \text { when } \theta=1, s_{k}=1 .\end{cases}
$$

In the case of fixed wall boundary conditions, these position probabilities are given by the same expressions, subject to the substitutions (4.16). So, as was to be expected, the position probabilities do depend on the position of the oscillator in the chain. 
For $p=1$ and $p=2$ it was possible to compute these position probabilities analytically, but for $p>2$ this becomes infeasible. On the other hand, since all coefficients $C_{r, \pm x_{K}, t}^{\theta, s}$ are known explicitly (in all cases and for any $p$ ), we can numerically compute all position probabilities. We will now examine the plots of some of these position probability distributions.

Let us first consider the case of periodic boundary conditions. The position probabilities are independent of $r$, as the system is completely symmetric, so let us take $r=1$. We will plot the values

$$
P\left(\theta, s, 1, \pm x_{K}\right), \quad K=0,1, \ldots, p
$$

for certain values of $\theta$ and $s$. In other words we plot the position probability distribution function when the system is in a fixed stationary state $w(\theta ; s)$. Let us consider an explicit example, say $n=4$ (four coupled oscillators) and $p=10$ (so each position operator has 21 distinct eigenvalues). We will plot the position probability distributions for the ground state (this is the state $w(0 ; 0, p, 0,0)=$ $w(0 ; 0,10,0,0))$ and for the most excited state (this is the state $w(1 ; 0,0,0, p-1)=w(1 ; 0,0,0,9)$ ). These distributions are given in Figure 4, for some $c$-values.

Let us make a number of observations on these distributions. When the system is in the ground state, the probability distribution function of each position operator is symmetric around its equilibrium position and unimodal. Of course it is also discrete (as we are working in finite-dimensional representations). As the coupling constant $c$ increases, the "peak" around the equilibrium position is sharper. In other words, a)s the coupling constant becomes larger, the oscillators are more likely to be close to their equilibrium position when the system is in its ground state.

When the system is in its most excited state, the position probabilities are quite different. The probability of finding the oscillator in its equilibrium position is zero. On the other hand, there are certain peaks away from the equilibrium position. As $c$ increases, these peaks are further away from the equilibrium position. In other words, as the coupling constant becomes larger, the oscillators are more likely to be further away from their equilibrium position when the system is in its most excited state.

Note that in this figure one also observes the fact that the range of the spectrum of the position operators is dependent on the coupling constant $c$. When talking about probabilities of being further or closer to the equilibrium position we regard this relative to the discrete spectrum of $2 p+1$ values (the middle one being the equilibrium position).

Let us now consider the case of fixed wall boundary conditions. The situation is rather different, as the position probabilities are depending on $r$. To see the $r$-dependence, we will plot position probability distribution functions for $r=1$ (the oscillator just next to the fixed wall) and for $r=3$ (an oscillator away from the wall). Again, we will plot the values

$$
P\left(\theta, s, r, \pm x_{K}\right), \quad K=0,1, \ldots, p
$$

for certain values of $\theta$ and $s$, i.e. when the system is in a fixed stationary state $w(\theta ; s)$. As an explicit example, take $n=6$ (six coupled oscillators) and $p=7$ (so each position operator has 15 distinct eigenvalues). We will again plot the position probability distributions for the ground state (this is the state $w(0 ; 0,0,0,0,0, p)=w(0 ; 0,0,0,0,0,7))$ and for the most excited state (this is the state $w(1 ; p-1,0,0,0,0,0)=w(1 ; 6,0,0,0,0,0))$. These distributions are given in Figure 5 for $r=1$ and in Figure 6 for $r=3$, for a number of $c$-values.

Let us again make a number of observations on these distributions. When the system is in the ground state, the probability distribution function of each position operator is symmetric around its equilibrium position and unimodal. For fixed $c>0$, if the oscillator is closer to the wall $(r=1)$ the peak of the distribution function around the equilibrium position is sharper than for an oscillator further away from the wall $(r=3)$. In other words, the oscillators close to the wall are closer to their equilibrium position than those further away from the wall. As the coupling constant $c$ 

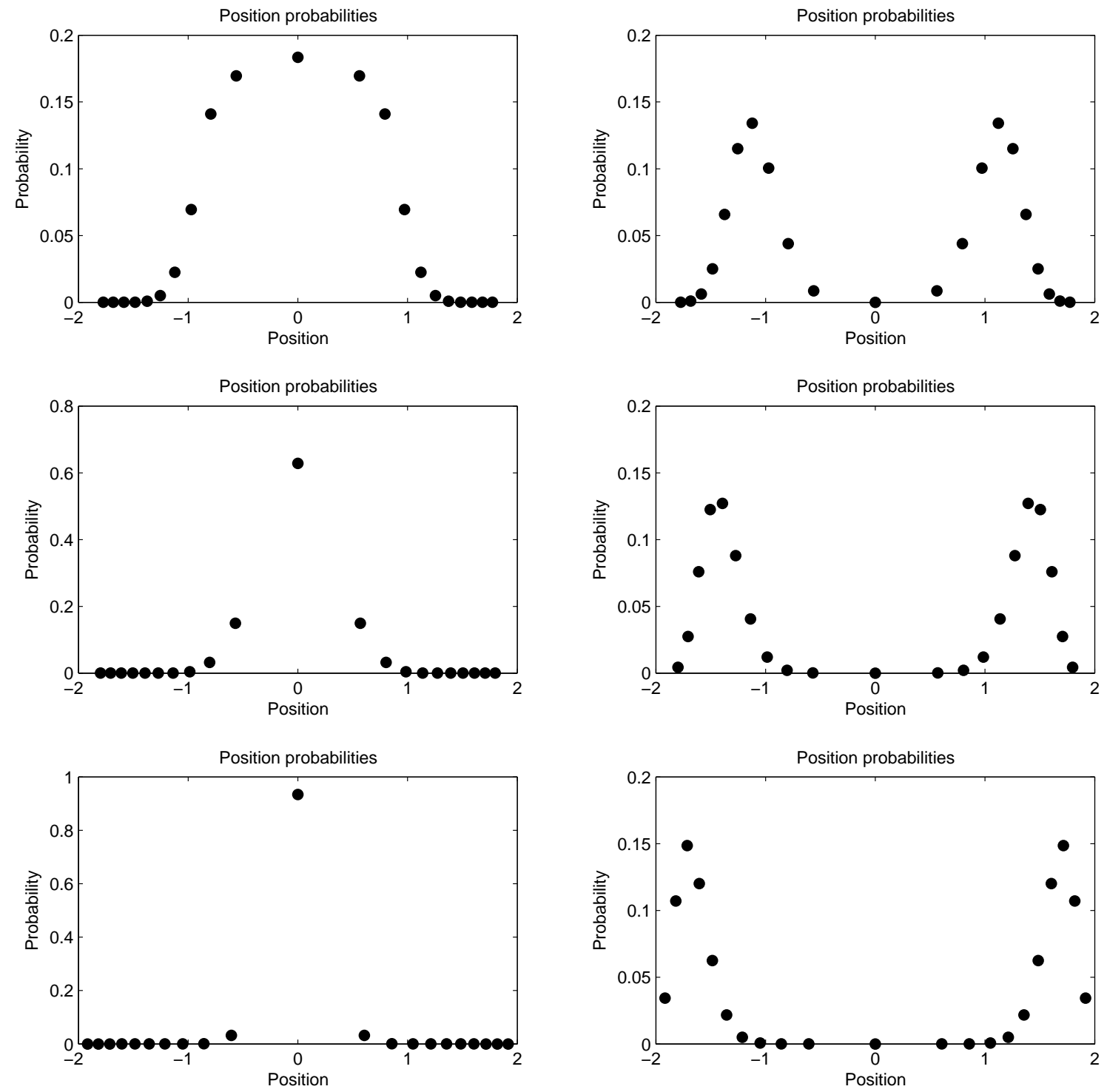

Figure 4: Position probability distribution function for the position operator $\hat{q}_{1}$, in the periodic boundary case, when $n=4$ and $p=10$. In the three rows, $c=0.1, c=0.4, c=0.8$. The plotted value is $P\left(\theta, s, 1, \pm x_{K}\right)$ for each of the 21 eigenvalues $\pm x_{K}(K=0,1, \ldots, 10)$ of $\hat{q}_{1}$. This is given for the case when the system is in the stationary state $w(\theta ; s)$ corresponding to the ground state (minimum energy) in the left column and in the right column when it is in the stationary state $w(\theta ; s)$ corresponding to the most excited state (maximum energy). 

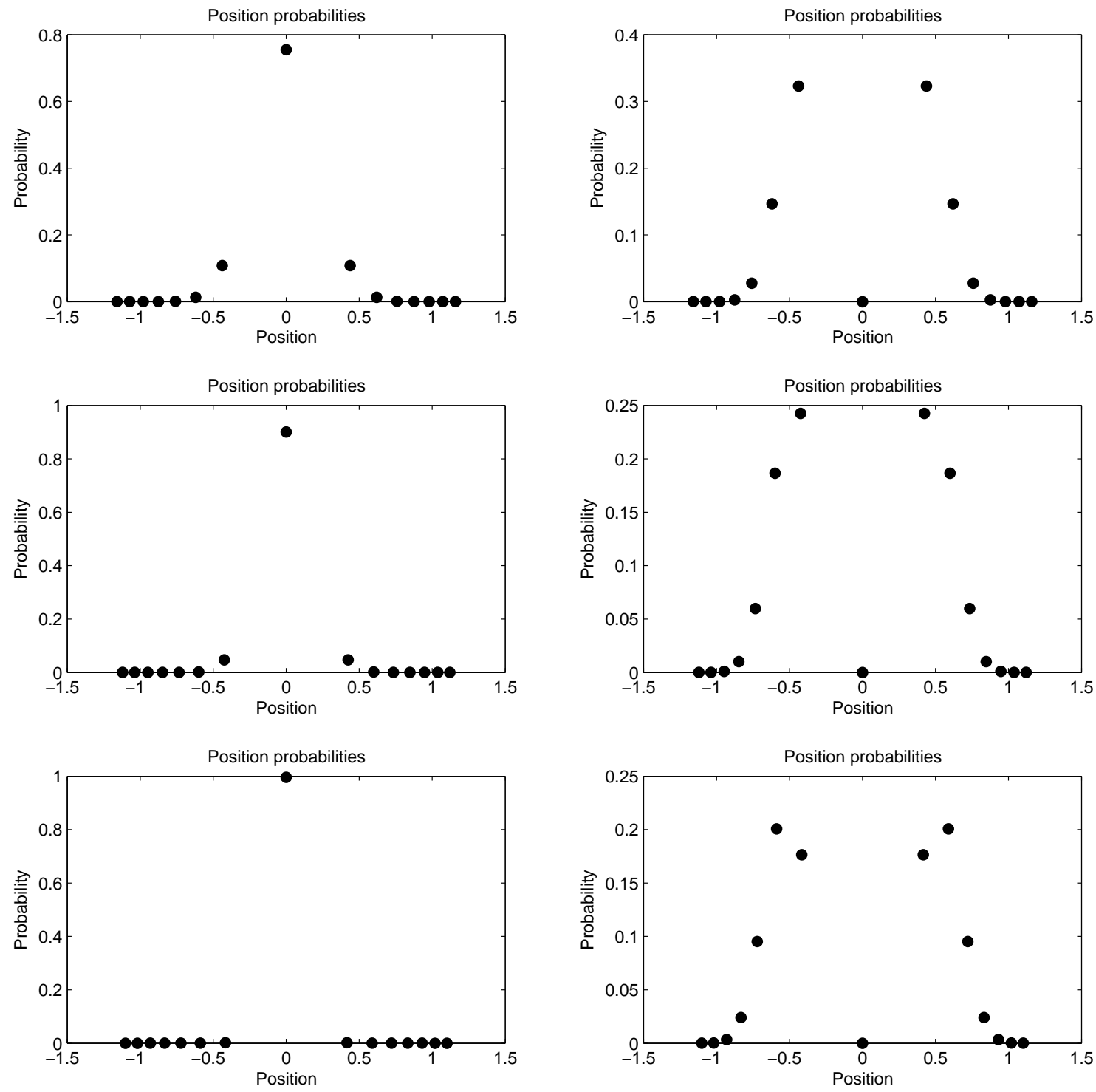

Figure 5: Position probability distribution function for the position operator $\hat{q}_{1}$, in the fixed wall boundary case, when $n=6$ and $p=7$. In the three rows, $c=0.05, c=0.2$, and $c=0.4$. The plotted value is $P\left(\theta, s, 1, \pm x_{K}\right)$ for each of the 15 eigenvalues $\pm x_{K}(K=0,1, \ldots, 7)$ of $\hat{q}_{1}$ (next to the wall). In the left column, this is given for the case when the system is in the stationary state $w(\theta ; s)$ corresponding to the ground state (minimum energy) and in the right column for the case when the system is in the stationary state $w(\theta ; s)$ corresponding to the most excited state (maximum energy). 

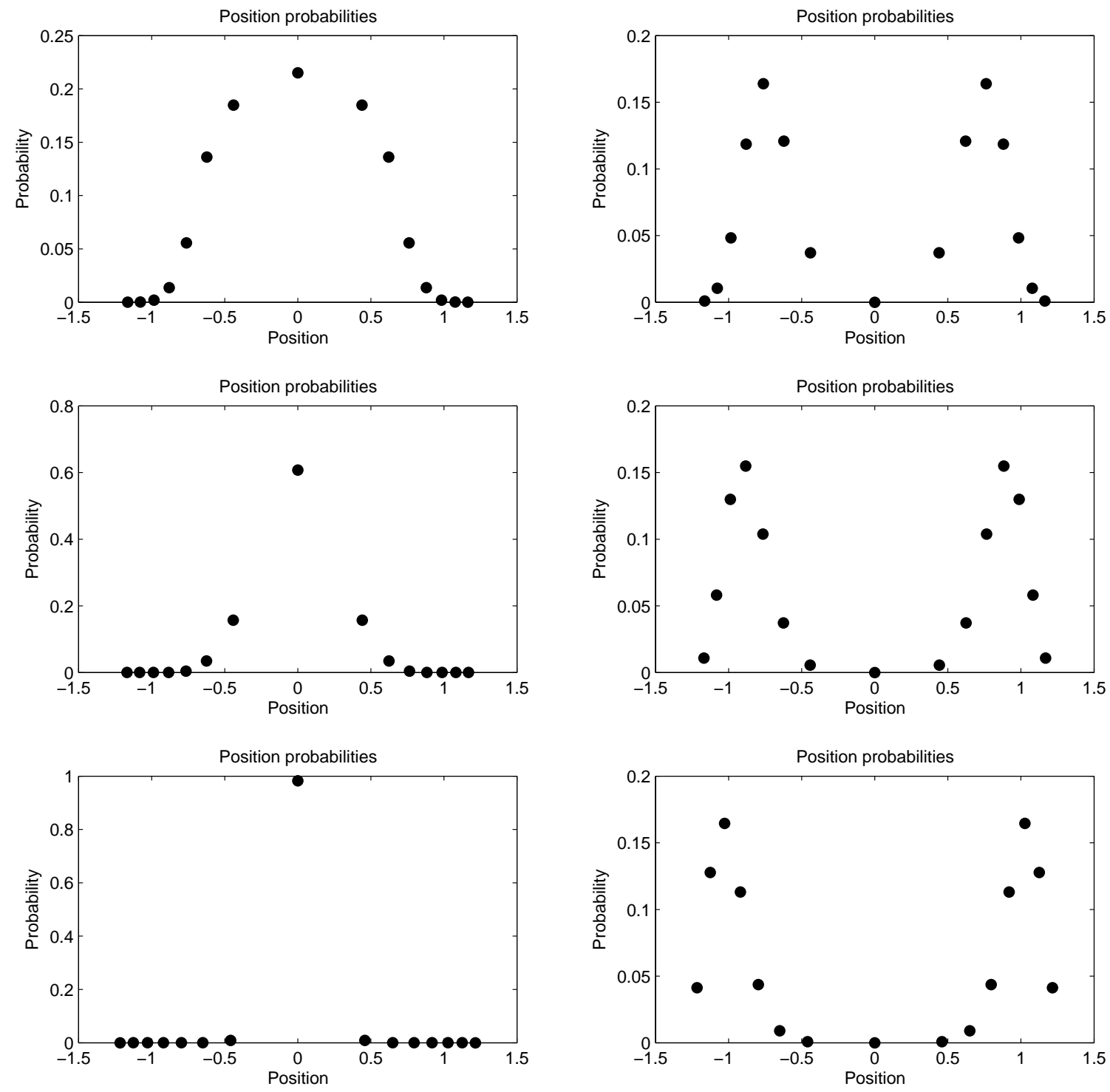

Figure 6: Position probability distribution function for the position operator $\hat{q}_{3}$, in the fixed wall boundary case, when $n=6$ and $p=7$. In the three rows, $c=0.05, c=0.2$, and $c=0.4$. The plotted value is $P\left(\theta, s, 1, \pm x_{K}\right)$ for each of the 15 eigenvalues $\pm x_{K}(K=0,1, \ldots, 7)$ of $\hat{q}_{1}$ (next to the wall). In the left column, this is given for the case when the system is in the stationary state $w(\theta ; s)$ corresponding to the ground state (minimum energy) and in the right column for the case when the system is in the stationary state $w(\theta ; s)$ corresponding to the most excited state (maximum energy). 
increases, the "peak" around the equilibrium position also becomes sharper, both for $r=1$ and $r=3$. In other words, as the coupling constant becomes larger, the oscillators are more likely to be close to their equilibrium position.

When the system is in its most excited state, the position probabilities are rather different. The probability of finding the oscillator in its equilibrium position is zero. For $c>0$, there are certain peaks away from the equilibrium position, both for $r=1$ and for $r=3$. Close to the wall $(r=1)$, these peaks are closer to zero than away from the wall $(r=3)$. So also in this most excited state, the oscillators close to the wall "oscillate less heavily" than those away from the wall. As $c$ increases, these peaks are further away from the equilibrium position, both for $r=1$ and for $r=3$. So the oscillators are more likely to be further away from their equilibrium position when the coupling constant increases.

\section{Conclusions}

We have examined properties of noncanonical solutions of two quantum systems: chains of coupled harmonic oscillators with periodic boundary conditions or with fixed wall boundary conditions. These new solutions arise from an approach as a WQS, allowing more classes of solutions than just the canonical one.

For the solutions examined here, the position and momentum operators are (odd) elements of the Lie superalgebra $\mathfrak{g l}(1 \mid n)$. The physical properties of the system then follow from the representations of $\mathfrak{g l}(1 \mid n)$ considered. Here, we have introduced the rather simple ladder representations $V(p)$, a class of unitary irreducible representations of $\mathfrak{g l}(1 \mid n)$ (or rather, of its compact form $\mathfrak{u}(1 \mid n)$ ). For these representations, we have determined the energy spectrum, which was rather easy due to the simple action of the Hamiltonian operator in the standard basis of $V(p)$. We have also determined the spectrum of the position operators; this task was more difficult because of the more complicated action of these operators in the basis of $V(p)$. The techniques developed in [14] allow to construct explicitly the eigenvectors of the position operators, for both systems under consideration.

The analysis of the spectrum of the position operators, and their probabilities when the system is in a certain stationary state, lead to interesting properties. The spectrum is discrete, centered around the equilibrium position; the number of possible position values depends on $p$ (it is $2 p+1$ ). The width of this discrete support depends on the coupling constant $c$, and - only in the case of fixed wall boundary conditions - on the order $r$ of the oscillator in the chain of $n$ oscillators. The position probability distributions, discussed in detail in the previous section, have properties similar to those of a classical system of coupled oscillators.

The current paper was still dealing with solutions arising from the Lie superalgebra $\mathfrak{g l}(1 \mid n)$, although a different class of representations was considered than in [7]. As indicated in a previous paper [7], also other types of solutions for the compatibility conditions exist, for example in terms

of the Lie superalgebra osp $(1 \mid 2 n)$. It would be interesting to investigate other solutions in terms of this orthosymplectic Lie superalgebra, even though the analysis is expected to be rather difficult.

\section{Acknowledgments}

N.I. Stoilova was supported by a project from the Fund for Scientific Research - Flanders (Belgium) and by project P6/02 of the Interuniversity Attraction Poles Programme (Belgian State - Belgian Science Policy). S. Lievens was also supported by project P6/02. 


\section{References}

[1] C. Cohen-Tannoudji, B. Diu and F. Laloë Quantum Mechanics (Wiley, New York, 1977), Vol. 1, complement JV.

[2] T.A. Brun and J.B. Hartle, Phys. Rev. D 60, 123503 (1999).

[3] K. Audenaert, J. Eisert, M.B. Plenio and R.F. Werner, Phys. Rev. A 66, 042327 (2002).

[4] J. Eisert and M.B. Plenio, Int. J. Quant. Inf. 1, 479-506 (2003).

[5] J.J. Halliwell, Phys. Rev. D 68, 025018 (2003).

[6] M.B. Plenio, J. Hartley and J. Eisert, New J. Phys. 6, 36 (2004).

[7] S. Lievens, N.I. Stoilova and J. Van der Jeugt, J. Math. Phys. 47, 113504 (2006).

[8] E. P. Wigner, Phys. Rev. 77, 711-712 (1950).

[9] A.H. Kamupingene, T.D. Palev and S.P. Tsavena, J. Math. Phys. 27, 2067-2075 (1986).

[10] T.D. Palev, J. Math. Phys. 23, 1778-1784 (1982); Czech. J. Phys., Sect. B 29, 91-98 (1979).

[11] V.G. Kac, Adv. Math. 26, 8-96 (1977).

[12] T.D. Palev, J. Math. Phys. 21, 1293-1298 (1980).

[13] R.C. King, N.I. Stoilova and J. Van der Jeugt, J. Phys. A 39, 5763-5785 (2006).

[14] S. Lievens, N.I. Stoilova and J. Van der Jeugt, J. Phys. A 40, 3869-3888 (2007). 